\title{
Evaluation of Transdermal Fentanyl Patch as Pre-Emptive Analgesia for Improvement of Postoperative Pain Relief in Patients Undergoing Major Abdominal Surgeries under General Anaesthesia
}

\author{
Saranya Rallabhandi1 ${ }^{1}$, Vivek Chakole ${ }^{2}$, Amol Singam ${ }^{3}$, Neeta Verma4 ${ }^{4}$, Anjali Modak ${ }^{5}$, \\ Karuna Taksande ${ }^{6}$, Sheetal Madavi7, Kashish Chaubey $^{8}$, Nikhil Bhalerao ${ }^{9}$ \\ 1, 2, 3, 4, 5, 6, 7, 8, 9 Department of Anaesthesiology, AVBRH, DMIMS (DU), \\ Sawangi Meghe, Wardha, Maharashtra, India.
}

\section{ABSTRACT}

\section{BACKGROUND}

An approach that is dynamic with a variety of treatment modalities is required for postoperative pain to obtain an optimal outcome which enhances comfort of the patient and facilitates the process of recovery. Pain which is poorly controlled is a major factor which contributes to a delay in discharge after most of the surgeries. Improvement of postoperative pain accelerates the ability of patients to resume their daily living activities. Various advanced techniques and novel analgesics have shown substantial potential which can improve various strategies for postoperative pain management. Transdermal drug delivery is one such methods which is not only promising but is also a non-invasive technique for the safe and effective administration of various pain medication. Systemic administration of analgesics can be done through this technique in a more advantageous way over existing technique. The requirement of needles and venous access are eliminated which makes its administration simpler, less invasive and more convenient. Fentanyl citrate is another synthetic narcotic with physicochemical characteristics i.e., high potency, skin compatibility, low molecular weight, and appropriate solubility that are suitable for rate controlled transdermal delivery. The primary objective is to assess the efficacy of transdermal fentanyl patch with respect to the number of rescue analgesic doses required in $24 \mathrm{hrs}$ of the study period. The secondary objective is to compare VAS, assess sedation and side effects associated with the patch, if any, in 24 hrs of the study period.

\section{METHODS}

60 patients of American Society of Anaesthesiologists I and II posted for elective major abdominal surgeries, were randomly allocated into two groups, 'Placebo' group ( $\mathrm{n}=30$ ) and 'Transdermal Fentanyl Patch' group ( $\mathrm{n}=30)$, receiving $25 \mathrm{mcg} /$ hr Duragesic patch applied on upper arm 10 hours before the surgery and were monitored for total number of rescue analgesic doses required, pain and sedation by VAS and RSS scale, respectively and side effects i.e., nausea, vomiting, pruritis and respiratory depression. The patients received inj. Paracetamol $1 \mathrm{gm}$ as rescue analgesia when the VAS score $>4$. The data was analysed by using Windows SPSS 17 version.

\section{RESULTS}

The requirement of number of rescue analgesic doses was less in patch group when compared to placebo $1.7 \pm 0.47$ vs $3.23 \pm 0.72$. The pain scores were less in fentanyl patch in comparison to placebo. Side effects were found to be comparable between the two groups.

\section{CONCLUSION}

Fentanyl transdermal patch of 25 microgram / hour applied 10 hours prior to surgery provides a safe, effective and non-invasive method of postoperative pain relief after major abdominal surgery.

\section{KEY WORDS}

Postoperative Pain, Transdermal Patch, Fentanyl
Corresponding Author: Dr. Saranya Rallabhandi. Department of Anaesthesiology, AVBRH, DMIMS (DU), Sawangi Meghe, Wardha, Maharashtra, India. E-mail: saruspicy@gmail.com

DOI: $10.14260 /$ jemds/2020/780

How to Cite This Article:

Rallabhandi S, Chakole V, Singam A, et al. Evaluation of transdermal fentanyl patch as pre-emptive analgesia for improvement of postoperative pain relief in patients undergoing major abdominal sugeries under general anaesthesia. J Evolution Med Dent Sci 2020;9(47):3556-3559, DOI: $10.14260 /$ jemds/2020/780

Submission 20-07-2020,

Peer Review 07-10-2020,

Acceptance 13-10-2020,

Published 23-11-2020.

Copyright (C) 2020 JEMDS. This is an open access article distributed under Creative Commons Attribution License [Attribution 4.0 International (CC BY 4.0)] 


\section{BACKGROUND}

Accurate management of pain is one of the most important challenges being faced by the health care providers. Uncontrollable acute pain in postoperative period results in long duration of hospital stay, frequent and unplanned hospital visits along with psychologic and physiologic effects hampering clinical outcome. Pain is amplified after surgical intervention because of central sensitization and hyperexcitability of neurons. ${ }^{1}$ Postoperative pain is experienced by more than $80 \%$ of patients undergoing surgery. ${ }^{2}$ Around $30-50 \%$ of Persistent Postsurgical Pain (PPP) originates from surgical intervention. The surgical treatment is carried out with an intention of better recovery for a good quality of life without any complications and sequelae. ${ }^{3}$ Pre-emptive pain relief is an antinociceptive treatment which prevents postoperative pain development by blocking afferent input formation. ${ }^{1}$ Traditionally, pain relief in perioperative period is achieved by the use of various opioid analgesics. However, prolonged opioid treatment for pain management in acute settings leads to a variety of perioperative side effects i.e., Post-Operative Nausea and Vomiting (PONV), drowsiness and sedation, pruritus, urinary retention, ileus, constipation, respiratory depression which can contribute to delay in hospital discharge and resumption of normal daily life. ${ }^{4}$ The transdermal route of drug delivery for analgesia is less invasive, simple, convenient and has various advantages over existing strategies like the elimination of requirement of needles and venous access, firstpass hepatic metabolism is bypassed and common barriers associated with oral analgesics i.e., nausea, vomiting and difficulty in swallowing are abolished. The drug absorption via the skin occurs through diffusion across the stratum corneum via a lipid-rich, intercellular pathway, though it possesses hindrance to passive drug delivery through transdermal route. Pain management with pharmacological agents with certain qualities i.e., low molecular weight, appropriate solubility in water and oil, an optimal partition coefficient between the membrane and the solution, and a low melting point are required for the easy drug transport across the skin. The physical and chemical characteristics of fentanyl i.e., a low molecular weight of $286 \mathrm{~g} / \mathrm{mol}$ high lipophilicity (octanolwater partition coefficient of 717) and optimal skin flux (approximately 1000 times that of morphine) allow it to be an optimal option for transdermal route of administration. ${ }^{5}$ Considering all the factors, the study was conducted to evaluate the efficacy of pre-emptive transdermal fentanyl patch in improvement of postoperative pain relief in patients undergoing major abdominal surgeries under general anaesthesia.

\section{METHODS}

The prospective, controlled randomised study proceeded from October 2019 to April 2020 in the Department of Anaesthesia, Jawaharlal Nehru Medical College, Acharya Vinoba Bhave Rural Hospital, Datta Meghe Institute of Medical Sciences, Sawangi (Meghe), Wardha, after taking due approval from the Ethical Committee. Consent was obtained in written form by 60 subjects of ASA I \& II between 20 - 70 years included in the study who were divided into 2 groups of 30 each, Computer generated random number table was used for randomisation. Group P (Placebo) who received micropore of the same size as the patch on the upper arm, 10 hours prior to surgery and Group TFP (Transdermal Fentanyl Patch) who received Duragesic transdermal fentanyl patch $25 \mathrm{mcg} / \mathrm{hr}$ on the upper arm, 10 prior to the surgery. Patients with allergy to opioids and on chronic analgesic use were excluded.

Open Epi Software was used for sample calculation by assuming an average VAS score of 3.79 and SD of 1.19, keeping power at $80 \%$ and confidence interval of $95 \%$ ( $\alpha$ error at $0.05)$, a sample size of 30 patients would be required to detect a minimum of $10 \%$ difference in the mean VAS score.

The enrolled patients underwent 120 - 180 minutes of elective major abdominal surgeries i.e., exploratory laparotomy, total abdominal hysterectomy, cholecystectomy, gastrectomy; under general anaesthesia. The patients NBM (Nil By Mouth) status of 8 hours was confirmed and 18G i.v catheter was secured and RL solution was started. After attaching all the monitors, baseline readings of Heart Rate (HR), Systolic Blood Pressure (SBP), Diastolic Blood Pressure (DBP), Oxygen Saturation (SpO2) and Electrocardiogram (ECG) were noted. Patients were premedicated with inj. midazolam $1 \mathrm{mg} / \mathrm{Kg}$, inj. fentanyl $2 \mathrm{mcg} / \mathrm{Kg}$, induced with inj. propofol $1-2 \mathrm{mg} / \mathrm{Kg}$, endotracheal intubation was facilitated with intubating dose of inj. vecuronium $0.1 \mathrm{mg} / \mathrm{Kg}$ and maintained by 1 - $2 \%$ sevoflurane with $50 \%$ oxygen and $50 \%$ nitrous oxide. Inj. Neostigmine $0.05 \mathrm{mg} / \mathrm{Kg}$ and glycopyrrolate $0.02 \mathrm{mg} / \mathrm{Kg}$ given at the end of surgery was used for reversal of neuromuscular blockade. Vitals like ECG, SPO2, SBP, DBP, ETCO2 were monitored intraoperatively throughout the surgery and in the recovery room for 2 hours postoperatively, after which the patients were shifted from the recovery room to postoperative ward. The patients were monitored for vitals, pain by Visual Analogue Scale (VAS), sedation by Ramsay Sedation Score (RSS) and adverse effects i.e., nausea, vomiting, pruritis, respiratory depression, every 4 hours for 24 hours from the time of arrival to postoperative ward. The patients received inj. paracetamol $1 \mathrm{gm}$ as rescue analgesic when the VAS $>4$.

- Visual Analogue Scale (VAS) - 1. Indicating no pain. 2. Probably no pain, 3. Mild discomfort. 4. Mild pain. 5. Mild to moderate pain. 6. Moderate pain, 7. Increased moderate pain. 8. Moderate to severe pain, 9. Severe pain. 10. Severe to excruciating pain.

- $\quad$ Ramsay Sedation Scale (RSS): 1. Anxious / restless or both 2. Co-operative, oriented and tranquil responding to command; 3. Brisk response to stimulus; 4. Sluggish response to stimulus; 5 . No response to any stimulus.

\section{Statistical Analysis}

The data was expressed as Mean \pm Standard Deviation. Statistical analysis was analysed by SPSS 17.0 version by using descriptive and inferential using student's unpaired $t$ test, chisquare test, Epi Info and GraphPad Prism 5.0 version. The statistically significant level was $\mathrm{p}<0.05$. 


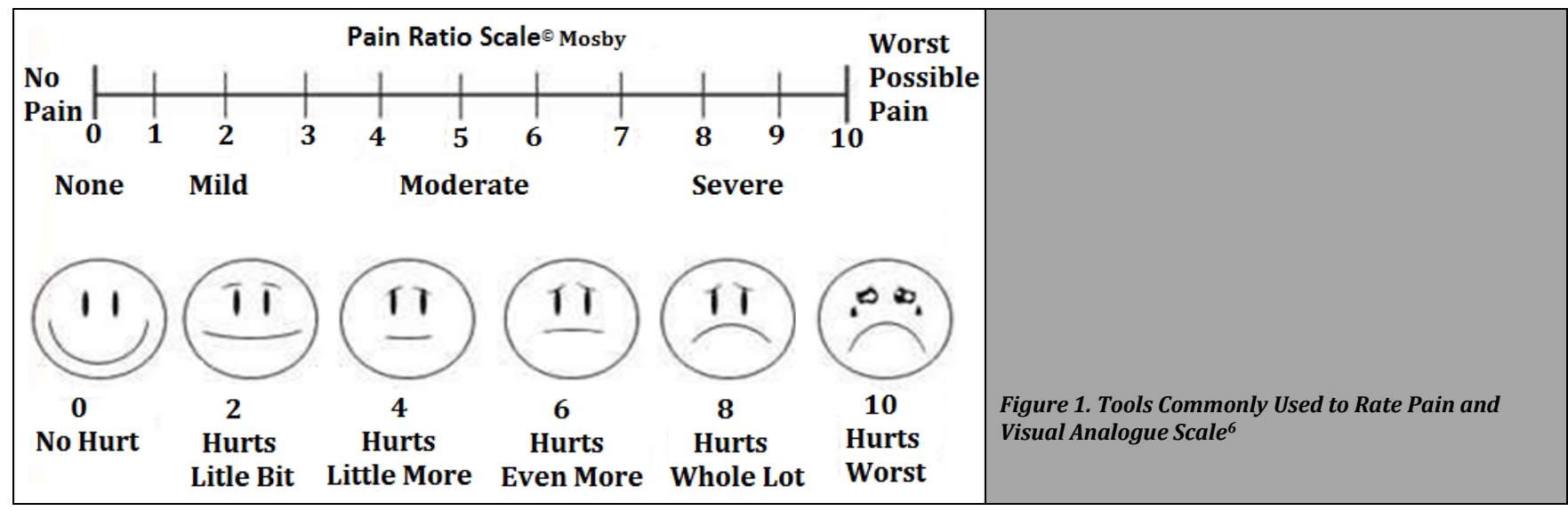

\section{RESULTS}

60 subjects divided into 2 groups with each group having 30 patients (Group P and Group TFP) were included in the study. The demographic data of both the groups i.e., mean age, mean weight of the patients, gender distribution and the surgery duration was statistically insignificant (Table 1).

\begin{tabular}{|cccc|}
\hline & Group P & Group TFP & P-Value \\
Mean age of patients (years) & $44.63 \pm 14.95$ & $40.90 \pm 14.00$ & $\mathrm{~T}=0.99 ;$ \\
$\mathrm{p}=0.32, \mathrm{NS}$ \\
Gender (male / female) & $15 / 15$ & $15 / 15$ & 1.00 \\
Mean weight of patients (Kg) & $58.23 \pm 4.24$ & $57.96 \pm 3.73$ & $\mathrm{t}=0.26 ;$ \\
$\begin{array}{c}\text { Mean duration of surgery } \\
\text { (minutes) }\end{array}$ & $159.0 \pm 7.23$ & $159.8 \pm 6.48$ & $\mathrm{~T}=0.45 ;$ \\
& & $\mathrm{p}=0.63, \mathrm{NS}$ \\
\hline Table 1. Demographic Data of Patients and Duration of Surgery \\
\hline
\end{tabular}

\begin{tabular}{|lccc|}
\hline & Group P & Group TFP & P-Value \\
\hline $\begin{array}{l}\text { Mean of the total number of } \\
\text { rescue analgesics in 24 hrs }\end{array}$ & $3.23 \pm 0.72$ & $1.7 \pm 0.47$ & $\mathrm{t}=9.75 ; \mathrm{p}=$ \\
& & $0.0001, \mathrm{~S}$ \\
\hline \multicolumn{3}{|c|}{ Table 2. Number of Rescue Analgesic Doses in 24 Hours } \\
\hline
\end{tabular}

The number of rescue analgesic doses required during 24 hours was less in fentanyl patch when compared to placebo (Table 2).

The mean pain intensity score observed over 24-hour postoperative period was significantly less in patients receiving transdermal fentanyl patch when compared to placebo (Table 3).

We can also suggest that patients with fentanyl patch had longer duration of analgesia when compared to placebo from looking at the mean pain scores over $24 \mathrm{hrs}$ of study.

\begin{tabular}{|ccccc|}
\hline $\begin{array}{c}\text { Time } \\
\text { Interval }\end{array}$ & Group P & Group TFP & t-Value & P-Value \\
\hline 0 hours & $3.2 \pm 1.2$ & $2.5 \pm 0.6$ & 3.2 & $0.006, \mathrm{~S}$ \\
4 hours & $2.5 \pm 1.2$ & $2.2 \pm 1.0$ & 1.05 & $0.29, \mathrm{NS}$ \\
\hline 8 hours & $3.4 \pm 1.2$ & $2.5 \pm 1.0$ & 3.15 & $0.002, \mathrm{~S}$ \\
12 hours & $2.6 \pm 1.0$ & $3.6 \pm 1.3$ & 3.34 & $0.001, \mathrm{~S}$ \\
\hline 16 hours & $2.4 \pm 1.0$ & $2.6 \pm 1.3$ & 0.66 & $0.5, \mathrm{NS}$ \\
\hline 20 hours & $3.4 \pm 1.4$ & $2.3 \pm 1.0$ & 3.50 & $0.001, \mathrm{~S}$ \\
\hline 24 hours & $2.4 \pm 1.4$ & $2.3 \pm 1.0$ & 0.31 & $0.75, \mathrm{NS}$ \\
\hline \multicolumn{5}{c}{ Table 3. Pain Scores over 24 Hours } \\
\hline
\end{tabular}

The mean sedation scores were found to be statistically significant in patients with fentanyl patch in comparison to placebo. The patients with patch were slightly more sedated than placebo group though none of the patients in patch group required any airway intervention (Table 4).

\begin{tabular}{|c|c|c|c|c|}
\hline $\begin{array}{c}\text { Time } \\
\text { Interval }\end{array}$ & Group P & Group TFP & t-Value & P-Value \\
\hline 0 hours & $1.5 \pm 1.2$ & $2.6 \pm 1.4$ & 3.26 & $0.002, \mathrm{~S}$ \\
\hline 4 hours & $1.4 \pm 1.5$ & $2.8 \pm 1.1$ & 4.12 & $0.002, S$ \\
\hline 8 hours & $2.1 \pm 1.0$ & $3.0 \pm 1.2$ & 3.15 & $0.002, \mathrm{~S}$ \\
\hline 12 hours & $1.6 \pm 1.4$ & $2.9 \pm 1.3$ & 3.72 & $0.0004, \mathrm{~S}$ \\
\hline 16 hours & $2.5 \pm 1.4$ & $3.2 \pm 1.3$ & 2.00 & $0.04, \mathrm{~S}$ \\
\hline 20 hours & $1.8 \pm 1.2$ & $3.0 \pm 1.2$ & 2.12 & $0.002, \mathrm{~S}$ \\
\hline 24 hours & $2.1 \pm 1.0$ & $2.6 \pm 1.4$ & 3.15 & $0.002, \mathrm{~S}$ \\
\hline
\end{tabular}

Adverse effects like nausea, vomiting, pruritis and respiratory depression were found to be comparable among both the groups which was statistically insignificant (Table 5).

\begin{tabular}{|ccccc|}
\hline Side Effects & Group P & Group TFP & $\chi 2$ - value & p-value \\
Nausea & $11(36.6 \%)$ & $8(26.6 \%)$ & 0.68 & 0.40, NS \\
Vomiting & $15(50 \%)$ & $9(30 \%)$ & 2.45 & 0.11, NS \\
Pruritis & $5(16.6 \%)$ & $7(23.3 \%)$ & 0.41 & 0.51, NS \\
$\begin{array}{c}\text { Respiratory } \\
\text { Depression }\end{array}$ & 0 & 0 & 0 & 0 \\
\hline \multicolumn{5}{c}{ Table 5. Adverse Effects among the Groups } \\
\hline \multicolumn{5}{c}{}
\end{tabular}

\section{DISCUSSION}

Post-surgical care has various components and effective pain management forms a major component. Acute pain includes post-surgical pain which occurs due to tissue trauma during surgery which results in an inflammatory reaction and afferent neuronal barrage initiation. Pain is a subjective phenomenon which is perceived only by the patient. Efficient postoperative pain control is an important factor for the safe discharge of the patient as pain that is inadequately treated can be a major cause of prolonged hospital stays. Thus, introduction of multimodal analgesia including opioids, non-opioids and regional blocks, either alone or in combination with other drugs have greatly improved pain management.

A novel, non-invasive strategy for the effective and safe administration of pain medication is transdermal drug delivery system which uses iontophoresis for the management of acute, moderate-to-severe postoperative pain.

Fentanyl binds to specific receptors within the CNS (Central Nervous System) at many sites which result in increase in pain threshold, altered reception of pain and inhibition of ascending pain pathways. In 1970, the suitability of delivery of fentanyl through the transdermal patch system was identified and has become one of the greatest commercial successes. $^{7}$ 
In our study, we found that the demographic characteristics of the study population like the mean age, mean weight, gender distribution and the surgery duration were comparable with no statistical significance between the two groups.

Patients with transdermal fentanyl patch had less pain and required less number of rescue analgesic doses, suggesting better analgesia with longer duration of action. Consistent with our results, Rowbotham et al, ${ }^{8}$ Sangineni et al, $^{9}$ and Hosseini et al, 7 found that application of transdermal fentanyl patch $10-12$ hrs. prior to the surgery showed a significant improvement in pain scores and it significantly reduced the morphine consumption in their studies.

In our study, we found the patients with fentanyl patch were slightly more sedated than placebo even though the patients did not require any airway intervention during the study.

Adverse effects like nausea, vomiting, erythema, pruritis, depression in respiration are common to opioids. In our study, we found that the side effects experienced by the patients i.e., nausea, vomiting, pruritis and respiratory depression were comparable with no statistical significance among the two groups.

Our results were supported by, Reinhart et al, ${ }^{10}$ studied on transdermal fentanyl patch plus intramuscular ketorolac for the treatment of postoperative pain relief and found that the incidence of pruritis was more with fentanyl patch even though the incidence of nausea and vomiting showed no difference between the groups.

Hosseini et al, ${ }^{7}$ studied on transdermal fentanyl patch for post abdominal laparotomy analgesia and found that nausea, vomiting, pruritis and respiratory depression were not significant between the groups.

Gupta et al, ${ }^{11}$ studied the post spinal effects of transdermal fentanyl patch in abdominal hysterectomy and found that fentanyl patch did not have any significant side effects.

\section{CONCLUSIONS}

Adequate postoperative pain management is one of the important factors for patient's discharge as inefficiently treated pain leads to prolonged hospital stays. Transdermal fentanyl patch is a non-invasive, self-contained drug delivery system with continuous parenteral administration of the drug. It has various advantages i.e., easy application, low infection risk, easy availability and cost effectiveness. Fentanyl patch 25 mcg / hr applied pre-emptively $10 \mathrm{hrs}$. prior to the surgery improves the quality of postoperative pain relief and is associated with comparable side effects in patients undergoing major abdominal surgeries under general anaesthesia.

Data sharing statement provided by the authors is available with the full text of this article at jemds.com.

Financial or other competing interests: None.

Disclosure forms provided by the authors are available with the full text of this article at jemds.com.

\section{REFERENCES}

[1] Beyaz SG, Bayar F, Erdem AF. Acute postoperative pain. J Anaesthe Clinic Res 2011;S7:002.

[2] Rawal N. Current issues in postoperative pain management. Eur J Anaesthesiol 2016;33(3):160-71.

[3] Luo J, Min S. Postoperative pain management in the postanaesthesia care unit: an update. J Pain Res 2017:10:2687-98.

[4] Elvir-Lazo OL, White PF. Postoperative pain management after ambulatory surgery: role of multimodal analgesia. Anaesthesiol Clin 2010;28(2):217-24.

[5] Power I. Fentanyl $\mathrm{HCl}$ iontophoretic transdermal system (ITS): clinical application of iontophoretic technology in the management of acute postoperative pain. Br J Anaesth 2007;98(1):4-11.

[6] Bushnik T. Visual analog scale. In: Kreutzer JS, DeLuca J, Caplan B, eds. Encyclopedia of clinical neuropsychology. New York, NY: Springer 2011.

[7] Hosseini H, Kargar S, Shiryazdi SM, et al. Fentanyl transdermal patch (Durogesic $₫$ D-TRANS) for post abdominal laparotomy analgesia: a double blind randomized study. Minerva Chir 2015;70(6):401-8.

[8] Rowbotham DJ, Wyld R, Peacock JE, et al. Transdermal fentanyl for the relief of pain after upper abdominal surgery. Br J Anaesth 1989;63:56-9.

[9] Sangineni KSD, Deolalkar A, Parakh SC, et al. Double blind randomized comparative study of transdermal fentanyl patch for post - operative pain relief in major abdominal surgery as a component of multimodal analgesic therapy. International Journal of Basic \& Clinical Pharmacology 2016;5(1):13-20.

[10] Reinhart DJ, Goldberg ME, Roth JV, et al. Transdermal fentanyl system plus im ketorolac for the treatment of postoperative pain. Canadian Journal of Anaesthesia 1997;44(4):377-84.

[11] Gupta R, Ahmed F, Chatterjee R, et al. Postspinal analgesic effect of transdermal fentanyl patch $(2.5 \mathrm{mg}$ and $5 \mathrm{mg}$ ) in abdominal hysterectomy: a randomized double-blind control study. Indian Journal of Pain 2016;30(2). 